\title{
Heart Failure and Malignancy: Implications of Chemotherapy and Radiation in the Pathogenesis of Cardiomyopathy in Cancer Treated Populations
}

\author{
Perry Wengrofsky ${ }^{1}$, Maya Srinivasan ${ }^{1}$, Haytham Aboushi ${ }^{1}$, Vaibhavi Solanki ${ }^{1}$, Inna Bukharovich ${ }^{2}$, Fadi Yacoub ${ }^{1}$, \\ Maria Poplawska ${ }^{1}$ and Samy I McFarlane ${ }^{1 *}$ \\ ${ }^{1}$ Department of Internal Medicine, State University of New York, Downstate Health Science University, Brooklyn, NY, USA \\ ${ }^{2}$ Division of Cardiovascular Disease, Department of Internal Medicine, NYC Health and \& Hospitals, Kings County, Brooklyn, NY, USA
}

Submission: November 23, 2020; Published: December 18, 2020

*Corresponding author: Samy I McFarlane, Department of Medicine, Division of Endocrinology Internal Medicine Residency Program Director State University of New York, Downstate Health Science University, 450 Clarkson Avenue, Box 50 Brooklyn, NY 11203-2098

\begin{abstract}
The field of Cardio-oncology is rapidly growing with significant advances in research leading to better understanding of the underlying pathogenesis with implications in the diagnosis and management of cancer-related cardiomyopathy.

Parallel to advancement in cardio-oncology is an increased awareness of the incidence of congestive heart failure and cardiomyopathy associated with malignancy. While specific cardiotoxic profiles exist for certain chemotherapeutic agents, there is increasing evidence of unexpected cardiotoxic side effects of some therapeutic modalities, combination chemo- and radiotherapy with large analyses identifying a strong association between malignancy and Takotsubo cardiomyopathy. Takotsubo Cardiomyopathy, also known as "broken-heart" syndrome or stress cardiomyopathy, is characterized by transient and reversible, regional or global, myocardial dysfunction without inciting ischemic perfusion defect from obstructive coronary artery disease. While direct causative pathophysiologic mechanisms continue to be investigated, much of the postulated pathways center on the high emotional and physical burdens of cancer and the related emotional stress associated with the diagnosis of cancer as well as the corporal effects of anti-neoplastic therapies, radiation, and oncologic surgery. In this manuscript we review the most current data in this rapidly emerging field highlighting the epidemiology, the postulated pathogenetic mechanisms as well as the current guidelines by major societies addressing malignancy -associated heart failure and cardiomyopathy, a rather complex disease entity with high morbidity and mortality.
\end{abstract}

Keywords: Heat Failure; Cardiomyopathy; Takotsubo Cardiomyopathy; Stress Cardiomyopathy; Cancer; Cardiotoxicity

\section{Introduction}

Among the greatest stories in modern medicine, research and advancements in diagnostics and therapeutics is the field of oncology, with continuously evolving management and diagnostic modalities, and establishment of regional and national centers of excellence in clinical investigation and care for patients with cancer, the second leading cause of death in the United States [1].

Highlighting the remarkable figures of increasing survival, reductions in mortality and annual incidence of cancer is a recent report with 2020 malignancy-associated morbidity and mortality projections by the American Cancer Society demonstrating the slowing of malignancy associated death and the notable gains in prevention and definitive treatment in cancer [2].

While malignancy survival, mortality, incidence, and prevalence figures have prominently improved since the 1970s, cardiovascular disease mortality risk, both in active cancer patients and cancer survivors, is prominently increased [3]. With the identification of malignancy associated cardiovascular morbidity and mortality, and known cardiotoxic profiles of cancer related therapies including chemotherapy, targeted antineoplastic agents, and radiotherapy, the field of cardio-oncology has become one of 
the most rapidly developing subspecialties in cardiology, with increasing numbers of fellowship training, clinical, and research programs, and dedicated scientific conferences, symposia, and publications [4-7].

Given the direct cardiomyocyte effects associated with chemotherapy, immunotherapy, other targeted antineoplastic agents, and radiotherapy, broad interest and attention has been given to cardiomyopathies and congestive heart failure (CHF) among the diverse cardiovascular pathologies identified in malignancy [8].

A particular cardiomyopathy and etiology of CHF of importance to cardiologists and oncologists is Takotsubo cardiomyopathy (TCM). TCM, also known as "broken-heart" syndrome or stress cardiomyopathy, is a syndrome of acute, transient, reversible, regional myocardial dysfunction with resulting ventricular hypokinesis, dyskinesis, or akinesis in the absence of underlying obstructive epicardial coronary artery disease, typically preceded by a physical or emotional stressor [9,10]. Large analyses of international TCM databases and registries have identified a strong association between malignancy and TCM [11]. Similar to investigations of pathophysiologic mechanisms of TCM in the absence of malignancy, postulations on TCM pathways in cancer patients center on identifiable emotional and physical triggers, noticeably abundant in malignancy, and include the emotional and mental stress of diagnosis and living chronically with cancer, and the physical stress associated with the symptoms of the disease itself and effects of therapies [12].

In this review, we present a comprehensive summary of the modern understanding of heart failure and cardiomyopathy through the lens of malignancy, analysis of epidemiology, cardiotoxic profiles of chemotherapy, radiotherapy, immunotherapy, and other targeted antineoplastic therapies, cardiovascular outcomes and diagnostic management strategies in the setting of direct therapy associated cardiomyopathy and in TCM, and consider current and future perspectives on areas of investigation in cardio-oncology.

\section{Epidemiology}

Historical trends and clinical observations have highlighted an increasingly apparent relationship between malignancy and cardiovascular disease. Among high income countries, cancer death rates exceed cardiovascular death rates. In the US, cardiovascular disease and malignancy are first and second leading causes of death, respectively. Therefore, from an epidemiological standpoint, identification of potential interactions between cancer and cardiovascular disease is of great importance $[1,13]$.

National measures of cardiovascular disease indicate $12.1 \%$ prevalence of adults with diagnosed heart disease, corresponding to approximately 30 million people with an annual mortality rate of approximately 200 people per 100,00 population [14]. Within cardiovascular pathologies, CHF prevalence has been estimated to be $2.2 \%$ among US adults above the age of 20 , with 1 -year and 5 -year mortality rates estimated at $22 \%$ and $42.3 \%$, respectively [15].

National measures of cancer-related incidence and survival point to overall improvements in morbidity and mortality, but highlight improving outcomes are not shared equally across malignancies or genders. Male and female mortality rates have similarly decreased since 1990, with 5-year survival rates as of 2015 at $67 \%$ [2]. However, since 2007, the incidence of new cancer diagnoses in males has declined to a rate of approximately 500 per 100,00 population, while the incidence rate among females has remained stable at approximately 400 per 100,000 population.

In parallel to the expanding research on the pathophysiology of cardiovascular disease in the setting of malignancy are data and epidemiologic profiles of cardiovascular complications in patients with cancer. Cardiovascular risk and burden of disease among cancer patients, both in terms of preexisting comorbidities (diabetes mellitus, smoking, etc.) and risk associated with specific malignancies and treatment modalities, have been increasingly identified as prevalent clinical vulnerabilities in malignancy $[16,17]$.

Among coexisting cardiovascular diseases in the US population of patients with lung, colorectal, breast, and prostate cancer, the prevalence of CHF exceeded the combined prevalence of cerebrovascular disease and myocardial infarction (MI) [17]. While HF prevalence was observed to be highest among Chinese cancer patients with hematologic malignancies, the hazard ratio of all-cause mortality was significantly increased for patients with CHF [16]. HF carries a lower 5-year survival rate than gender specific malignancies, such as prostate cancer in men and breast cancer in women.

Along with being at an increased risk for developing cancer, patients with CHF later diagnosed with malignancy have been shown to have higher all-cause mortality demonstrating an additive effect $[18,19]$. Epidemiology of HF and cardiomyopathy associated with chemotherapy and targeted antineoplastic therapies has been well documented in literature and has prompted major national and international cardiology and oncology societies to develop dedicated guidelines for the evaluation and management of cardiomyopathy and CHF attributed to malignancy and chemotherapy, or incorporate recommendations on chemotherapy induced CHF and cardiomyopathy into larger CHF guidelines [20-22]. Historically, anthracyclines and human epidermal growth factor receptor 2 (Her2) inhibitors have been commonly cited for their potent cardiotoxicity, with adjuvant use of these agents increasing the overall risk of cardiotoxicity [2325].

Moreover, in patients presenting with mild to end stage $\mathrm{CHF}$, or in those who are clinically asymptomatic but with clinical evidence of cardiomyopathy, doxorubicin, other anthracyclines, 
and non-anthracycline chemotherapeutic and anti-neoplastic agents were implicated as the etiology of up to $3 \%$ of initially unexplained cardiomyopathies [26,27].

Cardiotoxic Chemotherapy, Immunotherapy, Antineoplastic Targeted Therapy, and Radiotherapy Cardiomyopathy

While anthracyclines and Her2 inhibitors have the most cardiotoxic profiles and are frequently implicated in the development of cardiomyopathy and CHF, multiple chemotherapies, immunotherapies, and targeted antineoplastic therapies have also been highlighted by major cardiology societies and in national and international CHF guidelines [22,28].

Since the release of the American and European CHF guidelines in 2013 and 2016, respectively, numerous guideline updates and position papers have pointed to additional information on the pathophysiology and importance of monitoring of ventricular dysfunction in previously and newly implicated cardiotoxic chemotherapeutic drugs [20,22,28-30]. While the management of malignancy continues to transition and incorporate increasingly novel targeted antineoplastic therapies, the extensive use of chemotherapy over the last 50 years has resulted in a large body of evidence specifically on cardiomyopathy and CHF, the most worrisome of cardiovascular complications of chemotherapy.

\section{Anthracyclines}

Since first observations of myocardial dysfunction were made in the 1970s, anthracyclines, particularly doxorubicin, have been shown to possess a potent dose-dependent cardiomyocyte toxicity profile resulting in left ventricular (LV) dysfunction and CHF $[20,23,31]$. The transition from asymptomatic ventricular dysfunction to overt symptomatic CHF is highly variable with often long latencies between anthracycline exposure and the clinical manifestations of cardiomyopathy, with similarly diverse cardiac structural and functional findings including LV wall thinning, chamber dilation, increased LV wall stress, reduced ejection fraction (EF), and diastolic dysfunction [23,32,33].

Current understanding of mechanisms of anthracycline induced cardiomyocyte injury center on the generation of toxic reactive oxygen species (ROS), inhibition of Topoisomerase $2 \beta$ (Top2ß) and the resulting breaks in DNA [23,34]. Doxorubicin increases oxidative stress in the cytoplasm and mitochondria via direct catalysis and formation of irreversible complexes with cardiolipin in the inner mitochondrial membrane susceptible to peroxidation, promoting cardiomyocyte death via caspase mediated apoptosis and disrupting mitochondrial ATP metabolism, further contributing to cardiomyocyte death through cytoplasmic and mitochondrial swelling and sarcomere lesions [34]. Top2ß, a DNA replication enzyme that is highly expressed in both highly proliferating cancer cells and active quiescent non-proliferating cells like cardiomyocytes, is inhibited by anthracyclines, resulting is double-stranded DNA breaks and activation of p53 mediated apoptosis pathways [23,35].

Anthracycline's dose dependent cardiotoxic profiles show similar dose dependent LV dysfunction rates, best displayed by wide incidence ranges of $3-5 \%$ and $18-48 \%$ for low and high dose doxorubicin, respectively [20].

Predisposing risk factors for anthacycline-induced cardiomyopathy include female gender, African-American heritage, age $>65$ years, kidney disease, and concurrent radiation therapy at or near the heart as seen in malignancies of the chest and mediastinum such as lymphoma, lung, and breast cancer $[20,23]$.

While there is high variability in onset of cardiomyopathy after anthracycline exposure, large retrospective analysis conducted by Cardinale et al. on the timing of anthracycline cardiotoxic manifestations, both symptomatic and asymptomatic, show $98 \%$ of cases of cardiomyopathy develop within a year of anthracycline exposure, with a median of 3.5 months $[23,36]$. The temporal relationship between anthracycline exposure and manifestations of cardiotoxicity has led to different approaches to classification. Early effects are classified as cardiomyopathic changes that develop within the first year of treatment, subcategorized as either acute (after single dose or course) or early-onset chronic progressive (within first year), with late-onset chronic progressive effects developing after the first year of treatment $[20,36,37]$. However, the timing and degree of physiologic and structural LV abnormalities are largely viewed as a continuous progressive cardiotoxicity with decline in LVEF where patients who are initially asymptomatic but with clinical manifestations and evidence of cardiomyopathy eventually develop symptoms $[20,38]$.

\section{Human Epidermal Growth Factor Receptor 2 (Her2) Inhibitors}

Her2 inhibitors, namely trastuzumab and more recently developed agents including pertuzumab, lapatinib, and adotrastuzumab emtansine, target Her2, a cell surface tyrosine kinase receptor that is overexpressed in up to a quarter of breast cancers, with Her2 positivity associated with a more aggressive malignancy course, reduced survival, and increased risk of recurrence [39]. While most commonly connected to breast cancer, Her2 overexpression has also been seen and studied in gastric, gastroesophageal, and bony metastases of prostate malignancies [40-42].

In the absence of concurrent anthracycline use, trastuzumab carries a considerably low risk of cardiotoxicity, with rates of asymptomatic systolic dysfunction and overt CHF occurring at 3.2 and $0.5 \%$, respectively [39]. When included in adjuvant anthracycline-based regimens, the rates of both asymptomatic reductions in ejection fraction and symptomatic $\mathrm{CHF}$ increase to 
4.0 and $18.6 \%$, respectively $[39,43]$. Each Her2 targeted therapy inhibits Her2 mediated pathways in different ways, but share a common cardiomyopathic pathophysiology of disrupting the cardiomyocyte homeostatic functions of Her2 signaling pathway, disturbing cardiomyocyte responses to hemodynamic stress, interfering with sarcomeric organization and hypertrophy, and triggering accumulation of cardiotoxic reactive oxygen species $[39,44,45]$.

In contrast to anthracyclines that are directly cardiotoxic causing cardiomyopathy thats is generally progressive and irreversible, Her2 inhibitors disrupt the cardioprotective functions of the Her2 pathway, with the overwhelming cases of newly developed cardiomyopathies and overt CHF being reversible $[39,46]$. Beyond antecedent exposure to anthracyclines, risk factors associated with trastuzumab related cardiotoxicity include age, cardiovascular comorbidities like hypertension and diabetes mellitus, and African American ethnicity [39,47,48].

\section{Additional Therapies}

Beyond the frequently implicated anthracyclines and Her2 inhibitors, numerous additional conventional chemotherapies, immunotherapies, and targeted therapies have been shown to induce myocardial dysfunction resulting in reversible and irreversible cardiomyocyte changes [20].

Vascular Endothelial Growth Factor (VEGF) signaling pathway inhibitors, both direct VEGF inhibitors like bevacizumab, and downstream signaling pathway tyrosine kinase inhibitors (TKIs) have exhibited varying rates of LV dysfunction and CHF $[43,49,50]$. VEGF pathway inhibitors have been approved and are under investigation in multiple malignancies, particularly solid tumors, including renal, lung, gastroesophageal, breast, cervical, ovarian, and gastrointestinal stromal cancers [50]. The incidence of $\mathrm{LV}$ dysfunction with bevacizumab is approximated at 1.6-4.0\%, with large variability in incidence and severity of HF (higher New York Heart Association [NYHA] classification) influenced by dose, underlying malignancy, and previous or concurrent use of other cardiotoxic chemotherapy [20,51]. Different cardiomyopathic mechanisms have been suggested, including thinning of ventricular walls, depressed contractility, and loss of cardioprotective function mediated by VEGF [52]. Among VEGF pathway TKIs, the relative risk of developing both all grade and high grade (NYHA III-IV) CHF, was similar between specific TKIs like axitinib and non-specific TKIs like sunitinib, sorafenib, vandetanib, and pazopanib, with a collective all grade CHF risk of 2.69 [20,53]. Prospective echocardiographic studies and large meta-analyses on sunitinib approximate a CHF incidence of $4.1 \%$ with relative risk of 1.8 compared to placebo, driven by a $9.7 \%$ incidence of LV dysfunction [49,54].

As VEGF pathway inhibitors are typically used in metastatic disease on patients with limited life expectancies that often undergo interruption and discontinuation of therapies, the degree of reversibility, approximated at $60-80 \%$, and overall prognosis associated with VEGF pathway inhibitor mediated cardiomyopathy and CHF is difficult to assess $[20,55]$.

Other conventional chemotherapies have been associated with cardiotoxicity, LV dysfunction, and CHF with wide ranging incidences and dose-dependent relationships [20]. While infrequent, the cardiomyopathy associated with alkylating agents such as cyclophosphamide, ifosfamide, and cisplatin is typically irreversible $[20,56]$. The risk CHF secondary to cyclophosphamide is dose-dependent, with LV systolic dysfunction occurring shortly after initial administration and irreversibility setting in at doses greater than $1.55 \mathrm{~g} / \mathrm{m}^{2} /$ day $[56,57]$. The incidence of cardiomyopathy in cyclophosphamide rangers from $7-28 \%$, showing a similar dose dependent relationship to ifosfamide, with pathophysiologic mechanisms based on gross pathology and autopsy suggesting therapy induced hemorrhagic cardiomyocyte necrosis, interstitial edema, fibrin deposition, subendocardial hemorrhage, and epicardial petechial lesions [20,49,56]. Beyond bolus and total dose, additional predisposing risk factors for the development of LV dysfunction include older age and concurrent or previous use of other chemotherapeutic agents and mediastinal radiation [57]. Cisplatin has been associated with a later onset of $\mathrm{LV}$ dysfunction, with the appearance of symptomatic CHF more common in patients with preexisting myocardial disease that is exacerbated by the high volumes of intravenous fluids administered to mitigate cisplatin related oto- and nephrotoxicity [20,56]. Furthermore, cisplatin mediated myocardial ischemia strongly contributes to the development of ischemic cardiomyopathy and CHF as opposed to direct cardiomyocyte toxicity [20].

Antimetabolites, such as 5-fluorouracil and capecitabine, and anti-microtubule agents, such as docetaxel and paclitaxel, have low cardiomyocyte toxicity profiles with rare instances of cardiomyopathy and CHF [58]. Cardiomyopathies associated with antimetabolites and anti-microtubules are exceedingly rare and manifest in patients exposed to more commonly implicated therapies like anthracyclines, trastuzumab, and cyclophosphamide, rendering assessment of the individualized cardiomyocyte toxicity profiles of these agents difficult [20]. Studies have hypothesized different mechanisms of antimetabolite mediated direct cardiomyocyte toxicity, including increased reactive oxygen species and toxic metabolite formation, Krebs cycle disruption, myocardial dysfunction and resulting apoptosis and necrosis from caspase-activation [58,59]. While the literature on the cardiomyocyte toxicity profiles of antimetabolites and antimicrotubules continues to evolve, the prominent multifactorial cardiac effects associated with these chemotherapeutic agents, including arrhythmogenesis, high grade conduction abnormalities, endothelial dysfunction, coronary vasospasm, platelet aggregation and thrombotic risk, contribute to the development of cardiomyopathy and CHF [58,60,61]. 
Two additional classes of cancer therapies with emerging evidence of cardiovascular toxicity include the BCR-ABL tyrosine kinase and proteasome inhibitors [20,62,63]. Tyrosine kinase enzymes have roles in myocardial, vascular, and metabolic physiology. Analysis of BCR-ABL tyrosine kinase inhibitors, including imatinib and newer formulations like nilotinib, dasatinib, bosutinib, and poratinib, have demonstrated an increased risk of adverse cardiovascular events, primarily progression of atherosclerosis and related complications, especially in those with pre-existing disease $[20,62,64]$. While early studies on imatinib raised concern for cardiomyopathic toxicity, subsequent research on imatinib and the other aforementioned BCR-ABL tyrosine kinase inhibitors have not demonstrated therapy related myocardial dysfunction, but implicate these agents in the development of other non-atherosclerotic cardiovascular complications, including pulmonary arterial hypertension and QT interval prolongation, [62,65,66]. Given the high protein turnover and proteasome activity in cardiomyocytes, proposed mechanisms of proteasome inhibitor associated cardiotoxicity, specifically carlfizomib and bortezomib, have centered on caspase mediated apoptosis and activation of the unfolded protein response, a signaling pathway accelerated by the accumulation of misfolded and unfolded proteins typically degraded by the proteasome [20,63,67]. Among multiple myeloma patients exposed to proteasome therapy, the incidence of cardiomyopathy and CHF among those treated with carfilzomib was considerably higher than those managed with bortezomib, likely secondary to robust proteasome inhibition and cardiomyocyte injury given the more potent irreversible interaction of carfilzomib at the chymotrypsin-like site of the 20 S proteasome $[20,63,68]$.

\section{Radiotherapy Cardiotoxicity and Cardiomyopathy}

Incidence and prevalence of radiation induced toxicity is difficult to establish due to several factors, most notably temporal delay between radiation therapy and onset of symptoms, previous or concurrent exposure to cardiotoxic therapies, and overall failure to properly attribute cardiac pathophysiologic complications to previous radiation treatment [20].

While diverse pathophysiologic mechanisms are proposed to drive the cardiotoxicity of radiotherapy, the constellation of these mechanisms appears to be a synergistic cardiotoxicity from the cumulative long term exposure to both chemotherapy and radiotherapy. Previous studies in patients exposed to both, particularly anthracyclines in malignancies like breast cancer and lymphoma, have significantly higher rates of cardiomyopathy than in patients treated with chemotherapy alone [20,32,69]. Radiotherapy cardiotoxicity is primarily driven by endothelial damage and resultant inflammation causing accelerated atherosclerosis, coronary artery disease, and myocardial ischemia $[69,70]$. Furthermore, a marked cytokine mediated inflammatory process that increases collagen deposition with a diffuse pattern of fibrosis involving the pericardium, myocardium, and conduction system has been noted $[69,71]$.
Beyond the direct myocardial effects of previous or concurrent chemo-, immuno-, and antineoplastic therapy, the cardiomyopathy resulting from radiation is a combination of fibrotic complications, including diastolic dysfunction secondary to LV stiffening, and chronic pericardial effusions and constrictive pericarditis $[69,72]$. Diastolic dysfunction arises from myocardial, perivascular, and pericellular fibrosis caused by micro-ischemia and alterations of ventricular distensibility and compliance $[69,73]$. Pericardial radiation injury causes neovascularization that furthers micro-ischemia, fibrosis, and inflammation, leading to disrupted pericardial venous and lymphatic drainage and a chronic exudative pericardial effusion [69]. While some cancer patients may develop rapidly accumulating or large volume malignant effusions presenting with cardiac tamponade, this is more typical of metastatic involvement of the pericardium or direct local invasion of mediastinal and thoracic malignancies [74,75]. Chronic inflammation and fibrosis of the pericardium results in constrictive pericarditis and restrictive cardiomyopathy, disrupting ventricular hemodynamics through normalization of right ventricle (RV) and LV pressures, and RV encroachment on LV diastole with impaired filling and output, and loss of inspiratory negative thoracic pressure assisting in biventricular filling [72].

\section{Pre-, Peri-, and Post-treatment Approach to Cardiotoxic Chemotherapy}

Given the wide cardiotoxic profiles of chemotherapeutic, immunotherapeutic, and targeted antineoplastic agents and risk of developing of asymptomatic cardiomyopathy and symptomatic CHF, especially in malignancies where multiple therapies are used simultaneously or in succession, a thorough and careful cardiovascular workup and assessment of cardiovascular risk factors and predisposing comorbidities is recommended in the clinical practice guidelines of the major American and European oncology organizations [21,29]. The 2016 American Society of Clinical Oncology (ACSO) guidelines [29] and the 2012 European Society for Medical Oncology (ESMO) guidelines [21] give specific recommendations regarding cardiotoxic risk stratification, specifically with anthracycline and Her2 inhibitor use, and general approaches and strategies for prevention and surveillance before, during, and after treatment.

In addition to screening for cardiovascular risk factors and predisposing comorbidities like smoking, obesity, hyperlipidemia, hypertension, and underlying coronary artery disease, all patients should undergo an echocardiogram to assess for pre-existing structural abnormalities and LV systolic or diastolic dysfunction. Regardless of comorbidities and preexisting coronary artery or structural heart disease, the ACSO (Table 1) and ESMO (Table 2) classify cancer patients as high risk for cardiotoxicity depending on the cumulative dosage of chemotherapy and radiotherapy, specifically anthracyclines and trastuzumab. Lower dosages of other therapeutic agents combined with radiotherapy result in the same high-risk categorization. 


\section{Journal of Cardiology \& Cardiovascular Therapy}

Table 1: Summary of ASCO Guidelines [29].

\begin{tabular}{|c|}
\hline High Risk for Cardiac Dysfunction: \\
\hline Treatment that includes: \\
\hline - High-dose anthracycline (doxorubicin $>250 \mathrm{mg} / \mathrm{m}^{2}$, epirubicin $>600 \mathrm{mg} / \mathrm{m}^{2}$ ) \\
\hline - High-dose radiotherapy where heart in treatment field (RT > 30 Gy) \\
\hline $\begin{array}{l}\text { - Low-dose anthracycline (doxorubicin }<250 \mathrm{mg} / \mathrm{m}^{2} \text {, epirubicin }<600 \mathrm{mg} / \mathrm{m}^{2} \text { ) in combination with low-dose radiotherapy (RT }<30 \text { Gy) where } \\
\text { heart in treatment field }\end{array}$ \\
\hline Treatment with low-dose anthracycline or trastuzumab alone and presence of: \\
\hline - Multiple cardiovascular risk factors (smoking, hypertension, diabetes, dyslipidemia, obesity) \\
\hline - Age $>60$ yo \\
\hline - Previous compromised cardiac function \\
\hline o Borderline preserved ejection fraction $50-55 \%$ \\
\hline o History of myocardial infarction \\
\hline o Moderate to severe valvular dysfunction \\
\hline Treatment with low-dose anthracycline followed by trastuzumab \\
\hline Preventative Strategies Before Therapy: \\
\hline Avoiding/minimizing use of cardiotoxic therapies \\
\hline Comprehensive cardiovascular risk assessment and baseline echocardiography \\
\hline Preventative Strategies During Therapy: \\
\hline Active screen/modification of cardiovascular risk factors \\
\hline Cardioprotective dexrazoxane, continuous infusion, or liposomal formulation of doxorubicin in patients receiving high dose anthracyclines \\
\hline Lower radiation doses and use of precise and tailored radiation fields with exclusion of cardiac region as much as possible \\
\hline - Deep-inspiration holding in patients with mediastinal tumors or breast cancer \\
\hline - Intensity-modulated radiotherapy with varying radiation energy level \\
\hline Preferred Surveillance and Monitoring of Cardiac Function During and After Therapy: \\
\hline Echocardiography in patients with signs or symptoms of cardiac dysfunction during or after completion of therapy \\
\hline - Echocardiography-derived strain imaging \\
\hline $\begin{array}{l}\text { - Cardiac magnetic resonance imaging (MRI) or multigated acquisition (MUGA) scan if echocardiography not available or poor image quality (MR } \\
\text { preferred) }\end{array}$ \\
\hline - Serum cardiac biomarkers (troponin, natriuretic peptides) \\
\hline Echocardiography in patients without signs of symptoms of cardiac dysfunction but considered to be at increased risk \\
\hline Routine echocardiography surveillance in patients with metastatic breast cancer continuing to receive trastuzumab indefinitely \\
\hline In patients at risk for cardiac dysfunction who have completed therapy \\
\hline Echocardiogr \\
\hline
\end{tabular}

Table 2: Summary of ESMO Guidelines [21].

High Risk for Cardiac Dysfunction:

Specified Cumulative Anthracycline Dosing

- Doxorubicin $>500 \mathrm{mg} / \mathrm{m}^{2}$

- Liposomal doxorubicin $>900 \mathrm{mg} / \mathrm{m}^{2}$

- Epirubicin $>720 \mathrm{mg} / \mathrm{m}^{2}$

- Mitoxantrone $>120 \mathrm{c}$ 


\section{Journal of Cardiology \& Cardiovascular Therapy}

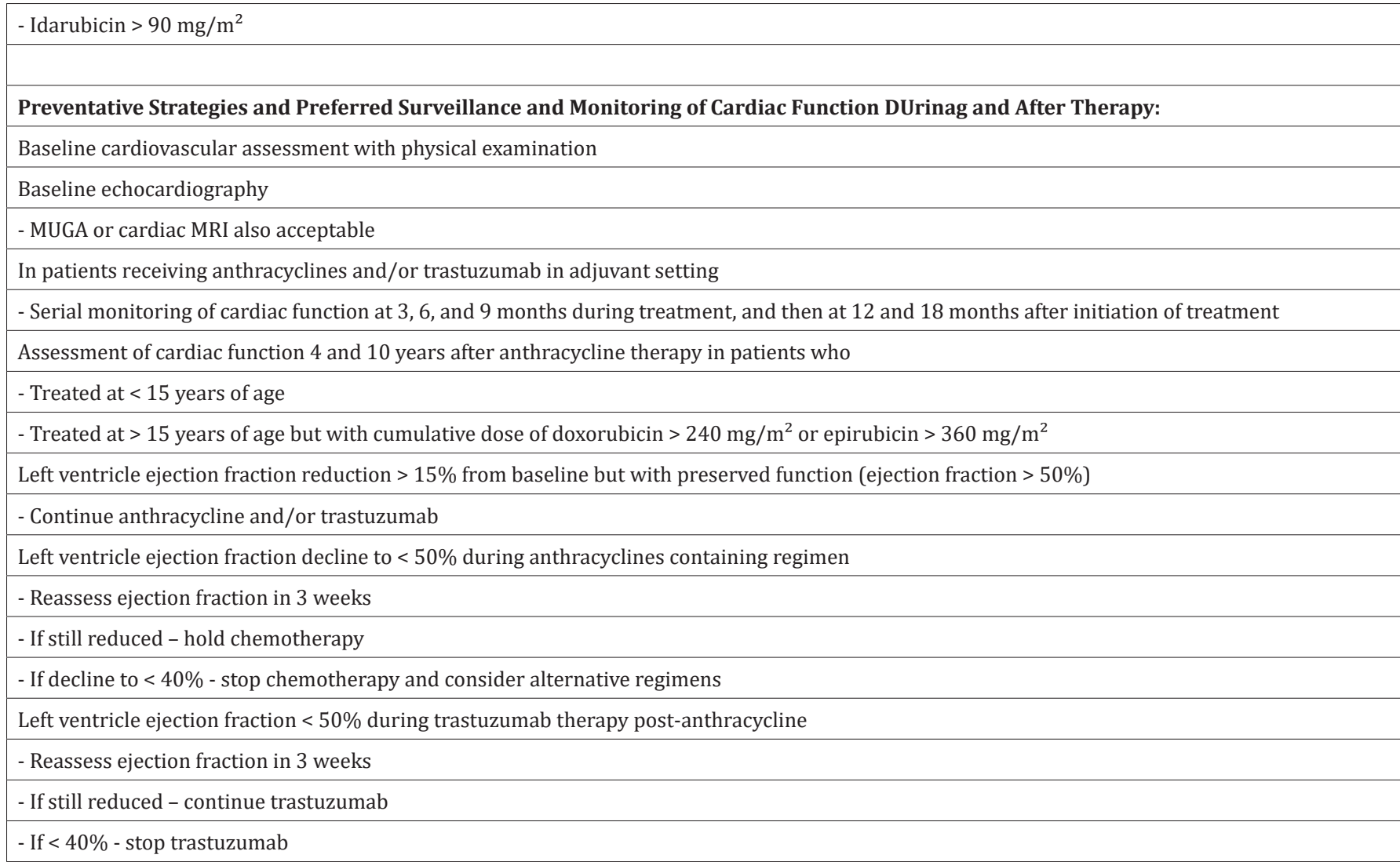

\section{Takotsubo Cardiomyopathy and Malignancy}

Recent data from international registries and large metaanalyses have identified a strong association between Takotsubo cardiomyopathy (TCM), malignancy and particularly poor outcomes $[11,76]$.

Epidemiologically, patients with TCM are more likely than age and gender matched peers to have a cancer diagnosis and with increased probability of having malignancy diagnosed in subsequent follow up [77]. Data from the InterTAK Registry, a multi-center collaboration across 9 countries, demonstrated a $16.6 \%$ prevalence of malignancy in patients with TCM [11].

Taken alongside other reviews, observational studies, metaanalyses, the prevalence of cancer in TCM has been approximated to be $1.3-25.5 \%$ [11,76-78]. Similar to investigations of pathophysiologic mechanisms of TCM in the absence of malignancy, postulations on TCM pathways in cancer patients center on identifiable emotional and physical triggers, noticeably abundant in malignancy, and include the emotional and mental stress of diagnosis and living chronically with cancer, and the physical stress associated with the symptoms of the disease itself and effects of therapies [12].

In the InterTAK Registry study from 2019, TCM in malignancy was more likely to have been caused by new pharmacological or procedural interventions than an emotional trigger and was associated with a higher in-hospital mortality and poorer longterm survival [11] (Figures $1 \& 2$ ).

\section{Current and Future Perspectives}

Given the primary and secondary rankings of cardiovascular disease and malignancy among annual national and international mortality, and the incidence of development of heart failure in patients with cancer, and vice versa, there has been an increasing focus on investigating the connections between cardiomyopathies and malignancy. Beyond known and postulated potential cardiotoxicities associated with specific chemotherapeutic, immunotherapeutic, and targeted antineoplastic agents, properties of particular hematologic and neuroendocrine malignancies increase the risk of cardiomyopathy, and highlight a bidirectional nature to cancer and heart failure [43].

Carcinoid heartdisease, araremanifestation of neuroendocrine tumors, is primarily characterized by endomyocardial fibrotic plaques and fibrotic remodeling of the endocardium $[43,79]$.

Fibroblast growth and fibrogenesis typically involves the valves, and given the efficient inactivation of neuroendocrine mediators in the pulmonary vasculature and liver, the resulting valvular stenosis or regurgitation is greatly influenced by the anatomic location of the tumor, with bronchial carcinoids resulting in rare mitral, aortic, and left ventricular dysfunction, or the presence of a right-to-left intracardiac shunt $[43,79,80]$. Tricuspid, pulmonic, and right ventricular carcinoid heart disease arises from gastrointestinal neuroendocrine tumors with metastases to liver that have significantly disrupted hepatic clearance of serotonin, tachykinin, and kallikrein, or rare primary 


\section{Journal of Cardiology \& Cardiovascular Therapy}

ovarian neuroendocrine tumors that avoid the portal venous system [79,81]. Myocardial metastasis of neuroendocrine cancerous cells is rare, and while direct tumor deposition and proliferation in the myocardium can contribute to systolic and diastolic dysfunction, right, left, or biventricular heart failure in the presence of neuroendocrine malignancies more typically arises from valvular involvement and resulting disruptions in normal physiology $[79,82]$.

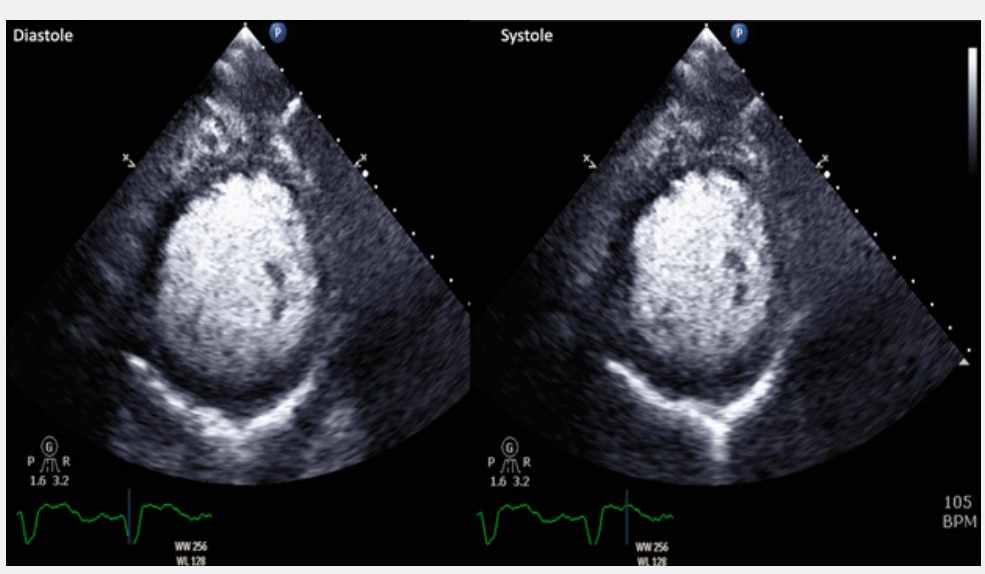

Figure 1: Parasternal short axis view of the left ventricle on transthoracic echocardiography in 68 year-old female patient with history of chronic lymphoid leukemia who had received 6 cycles of CHOP (cyclophosphamide, doxorubicin hydrochloride [hydroxydaunorubicin], vincristine sulfate [Oncovin], prednisone] before developing HF. Echocardiography revealed reduced ejection fraction (EF) to $30-35 \%$ with global LV hypokinesis, highlighted by minimal change in internal diameter and difference between end diastolic volume (left) and end systolic systole volume (right).
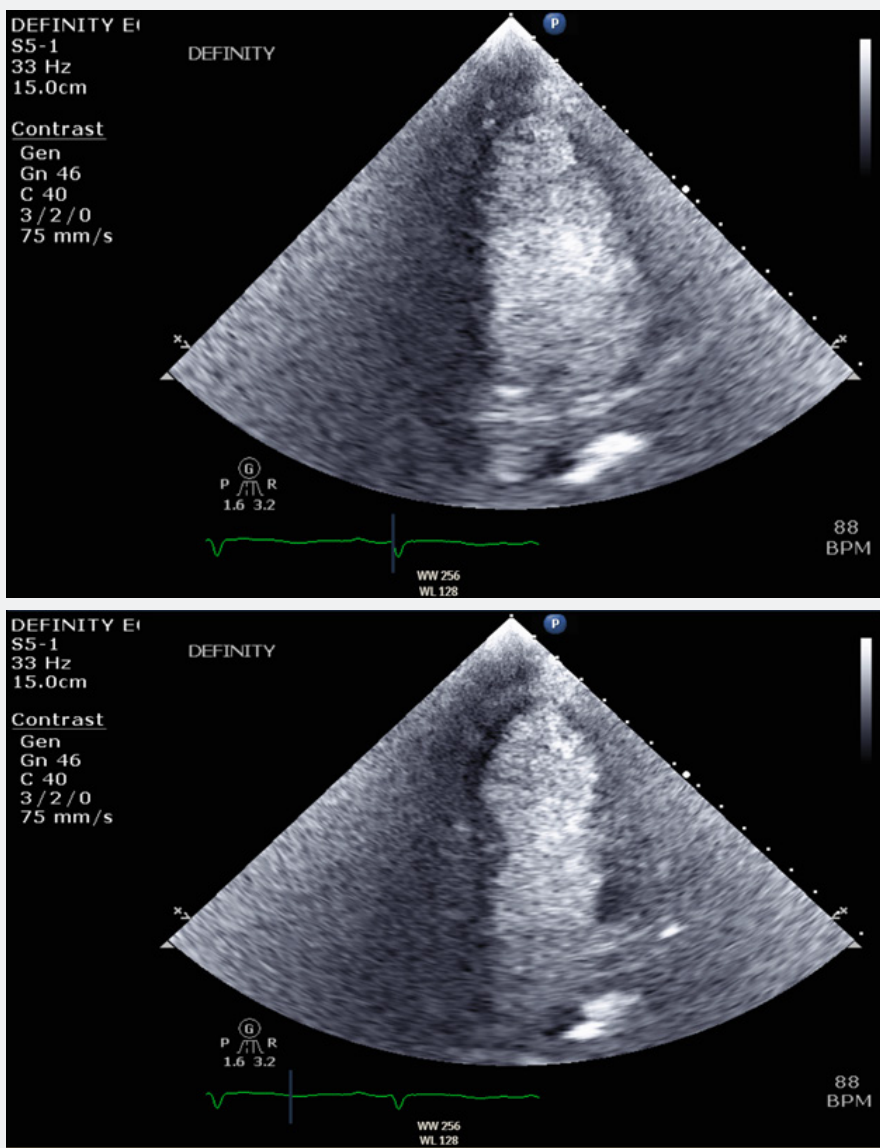

Figure 2: Transthoracic echocardiography apical view of LV with Definity contrast enhancement of diastole (top) and systole (bottom) demonstrating basal LV contraction with apical hypokinesis and resulting apical ballooning. 
In hematologic malignancies and plasma cell dyscrasias like chronic lymphocytic leukemia, macroglobulinemia, nonlymphoplasmacytic lymphoma, monoclonal gammopathy of unknown significance, smolder myeloma, and multiple myeloma, cardiac deposition of light chain immunoglobins and $\mathrm{AL}$ amyloidosis in the myocardium and valves contributes to the development of restrictive cardiomyopathy and heart failure from cardiac amyloidosis $[43,83]$. In cardiac amyloidosis, amyloid fibril infiltration causes increased wall thickness with restrictive physiology, diastolic dysfunction, smaller end-diastolic volumes, and despite preserved ejection fractions and systolic function, overall lower stroke volumes and cardiac output [84].

More recent early experimental work on cardiotoxic oncometabolites has elucidated additional pathophysiologic pathways of cardiomyopathy in cancer [43]. In acute myeloid leukemia, alterations in genetic and global expression of isocitrate dehydrogenase 1 and 2 has been shown in retrospective observational studies to be associated with a higher prevalence of coronary artery disease, and to mechanistically exacerbate doxorubicin mediated cardiotoxicity [85].

\section{Conclusion}

The discipline of cardio-oncology continues to emerge with the expansion of dedicated subspecialty fellowship programs and cancer and cardiovascular institution based cardio-oncology centers and services. As cardiologists and oncologists learn more and more about these maladies and the evolving understanding of connections between them, the clinical care of patients with asymptomatic cardiomyopathy, symptomatic congestive heart failure, and malignancy will be continually refined. It is unquestionably an exciting and privileged time for cardiooncology, and through continued collaboration, the longitudinal and personalized cardiac and cancer care of these high-risk patients will mature and improve.

Acknowledgement: This work is supported, in part, by the efforts of Dr. Moro O. Salifu MD, MPH, MBA, MACP, Professor and Chairman of Medicine through NIMHD Grant number S21MD012474.

\section{References}

1. Kochanek KD, Murphy SL, Xu J, Arias E (2019) Deaths: Final Data for 2017. National Vital Statistics Report; 68(9). Hyattsville, MD: National Center for Health Statistics.

2. Siegel RL, Miller KD, Jemal A (2020) Cancer Statistics, 2020. CA Cancer J Clin 0: 1-24.

3. Sturgeon KM, Deng L, Bluethmann SM, Zhou S, Trifiletti DM, et al. (2019) A Population-based Study of Cardiovascular Disease Mortality Risk in US Cancer Patients. Eur Heart J 40(48): 3889-3897.

4. Hong RA, Iimura T, Sumida KN, Eager RM (2010) Cardio-oncology/ onco-cardiology. Clin Cardiol 33(12): 733-777.

5. Snipelisky D, Park JY, Lerman A, Mulvagh S, Lin G, et al. (2017) How to Develop a Cardio-oncology Clinic. Heart Fail Clin 13(2): 347-359.
6. Fradley MG (2019) Curr Treat Options Cardiovasc Med 21(6): 27.

7. Cardio-Oncology Section News. American College of Cardiology.

8. Anker MS, Lena A, Hadzibegovic S, Belenkov Y, Bergler-Klein J, et al. (2018) Modern-day Cardio-oncology: A Report from the 'Heart Failure and World Congress on Acute Heart Failure 2018'. ESC Heart Fail 5(6): 1083-1091.

9. Ono R, Falcao LM (2016) Takotsubo Cardiomyopathy Systematic Review: Pathophysiologic Process, Clinical Presentation and Diagnostic Approach to Takotsubo Cardiomyopathy. Int J Cardiol 209: 196-205.

10. Pelliccia F, Kaski JC, Crea F, Camici PG (2017) Pathophysiology of Takotsubo Syndrome. Circulation 135(24): 2426-2441.

11. Cammann VL, Sarcon A, Ding KJ, Seifert B, Kato K, et al. (2019) Clinical Features and Outcomes of Patients with Malignancy and Takotsubo Syndrome: Observations from the International Takotsubo Registry. J Am Heart Assoc 8(15): e010881.

12. Desai A, Noor A, Joshi S, Kim AS (2019) Takotsubo Cardiomyopathy in Cancer Patients. Cardio-Oncology 5: 7.

13. Dagenais GR, Leong DP, Rangarajan S, et al. (2019) Variations in Common Diseases, Hospital Admissions, and Deaths in Middle-aged Adults in 21 Countries from Five Countries (PURE): A Prospective Cohort Study. Lancet pii: S0140-6736(19)32007-0.

14. Heart Disease. National Center for Health Statistics. Centers for Disease Control and Prevention.

15. Benjamin EJ, Muntner P, Alonso A, Bittencourt MS, Callaway CW, et al. (2019) Heart Disease and Stroke Statistis-2019 Update: A Report from the American Heart Association. Circulation 139(10): e56-e528.

16. Liu D, Ma Z, Yang J, Zhao M, Ao H, et al. (2019) Prevalence and Prognosis Significance of Cardiovascular Disease in Cancer Patients: A Population-based Study. Aging (Albany NY) 11(18): 7948-7960.

17. Edwards BK, Noone AM, Mariotto AB, Simard EP, Boscoe FP, et al. (2014) Annual Report to the National on the Status of Cancer, 19752010, Featuring Prevalence of Comorbidity and Impact on Survival Among Persons with Lung, Colorectal, Breast, or Prostate Cancer. Cancer 120(9): 1290-1314.

18. Ameri P, Canepa M, Anker MS, Belenkov Y, Bergler-Klein J, et al. (2018) Cancer Diagnosis in Patients with Heart Failure: Epidemiology, Clinical Implications and Gaps in Knowledge. Eur J Heart Fail 20(5): 879-887.

19. Banke A, Schou M, Videbaek L, Møller JE, Torp-Pedersen C, et al. (2016) Incidence of Cancer in Patients with Chronic Heart Failure: A Longterm Follow-up Study. Eur J Heart Fail. 2016; 18(3): 260-266.

20. Zamorano JL, Lancellotti P, Rodriguez Munoz D, Aboyans V, Asteggiano R, et al. (2016) 2016 ESC Position Paper on Cancer Treatments and Cardiovascular Toxicity Developed Under the Auspices of the ESC Committee for Practice Guidelines: The Task Force for Cancer Treatments and Cardiovascular Toxicity of the European Society of Cardiology (ESC). Eur Heart J 37(36): 2768-2801.

21. Curigliano G, Cardinale D, Suter T, Plataniotis G, Azambuja E de, et al. (2012) Cardiovascular Toxicity Induced by Chemotherapy, Targeted Agents and Radiotherapy: ESMO Clinical Practice Guidelines. Ann Oncol 23 Suppl 7: vii155-166.

22. Yancy CW, Jessup M, Bozkurt B, Butler J, Casey Jr DE, et al. (2013) 2013 ACCF/AHA Guideline for the Management of Heart Failure: A Report of the American College of Cardiology Foundation/American Heart Association Task Force on Practice Guidelines. J Am Coll Cardiol 62(16): e147-e239.

23. Henriksen PA (2018) Anthracycline Cardiotoxicity: An Update on Mechanisms, Monitoring, and Prevention. Heart 104(12): 971-977. 
24. Ponde NF, Lambertini M, de Azambuja E (2016) Twenty Years of antiHER2 Therapy-associated Cardiotoxicity. ESMO Open 1(4): e000073.

25. Ezaz G, Long JB, Gross CP, Chen J (2014) Risk Prediction Model for Heart Failure and Cardiomyopathy after Adjuvant Trastuzumab Therapy for Breast Cancer. J Am Heart Assoc 3(1): e000472.

26. Felker GM, Thompson RE, Hare JM, Hruban RH, Clemetson DE, et al. (2000) Underlying Causes and Long-term Survival in Patients with Initially Unexplained Cardiomyopathy. N Engl J Med 342(15): 1077 1084.

27. Araujo-Gutierrez R, Ibarra-Cortez SH, Estep JD, Bhimaraj A, Guha A, et al. (2018) Incidence and Outcomes of Cancer Treatment-related Cardiomyopathy Among Referrals for Advanced Heart Failure. CardioOncology 4(3).

28. Ponikowski P, Voors AA, Anker SD, Bueno H, Cleland JGF, et al. (2016) 2016 ESC Guidelines for the Diagnosis and Treatment of Acute and Chronic Heart Failure: The Task Force for the Diagnosis and Treatment of Acute and Chronic Heart Failure of the European Society of Cardiology (ESC). Developed with the Special Contribution of the Heart Failure Association (HFA) of the ESC. Eur J Heart Fail 18(8): 891-975.

29. Armenian SH, Lacchetti C, Barac A, Carver J, Constine LS, et al. (2017) Prevention and Monitoring of Cardiac Dysfunction in Survivors of Adult Cancers: American Society of Clinical Oncology Clinical Practice Guidelines. J Clin Oncol 35(8): 893-911.

30. Plana JC, Galderisi M, Barac A, Ewer MS, Ky B, et al. (2014) Expert Consensus for Multimodality Imaging Evaluation of Adult Patients During and After Cancer Therapy: A Report from the American Society of Echocardiography and the European Association of Cardiovascular Imaging. J Am Soc Echocardiogr 27(9): 911-939.

31. Gianni L, Herman EH, Lipshultz SE, Minotti G, Sarvazyan N, et al. (2008) Anthracycline Cardiotoxicity: From Bench to Bedside. J Clin Oncol 26(22): 3777-3784.

32. Armenian SH, Hudson MM, Mulder RL, Chen MH, Constine LS, et al. (2015) Recommendations for Cardiomyopathy Surveillance for Survivors of Childhood Cancer: A Report from the International Late Effects of Childhood Cancer Guideline Harmonization Group. Lancet Oncol 16(3): e123-e136.

33. Boyd A, Stoodley P, Richards D, Hui R, Harnett P, et al. (2017) Anthracyclines Induce Early Changes in Left Ventricular Systolic and Diastolic Dysfunction: A Single Centre Study. PLoS One 12(4): e0175544.

34. Nebigil CG, Desaubry L (2018) Updates in Anthracycline-Mediated Cardiotoxicity. Front Pharmacol 9: 1262.

35. McGowan JV, Chung R, Maulik A, Piotrowska I, Walker JM, et al. (2017) Anthracycline Chemotherapy and Cardiotoxicity. Cardiovasc Drugs Ther 31(1): 63-75.

36. Cardinale D, Colombo A, Bacchiani G, Tedeschi I, Meroni CA, et al. (2015) Early Detection of Anthracycline Cardiotoxicity and Improvement with Heart Failure Therapy. Circulation 131(22): 1981-1988.

37. Lipshultz SE, Alvarez JA, Scully RE (2008) Anthracycline Associated Cardiotoxicity in Survivors of Childhood Cancer. Heart 94(4): 525-533.

38. Eschenhagen T, Force T, Ewer MS, de Keulenaer GW, Suter TM, et al. (2011) Cardiovascular Side Effects of Cancer Therapies: A Position Statement from the Heart Failure Association of the European Society of Cardiology. Eur J Heart Fail 13(1): 1-10.

39. Copeland-Halperin RS, Liu JE, Yu AF (2019) Cardiotoxicity of HER2targeted Therapies. Curr Opin Cardiol 34(4): 451-458.

40. Kanayama K, Imai H, Usugi E, Shiraishi T, Hirokawa YS, et al. (2018) Association of HER2 Gene Amplification and Tumor Progression in Early Gastric Cancer. Virchows Arch 473(5): 559-565.
41. Grillo F, Fassan M, Sarocchi F et al. (2016) HER2 Heterogeneity in Gastric/gastroesophageal Cancers: From Benchside to Practice. World J Gastroenterol 22(26): 5879-5878.

42. Day KC, Lorenzatti Hiles G, Kozminsky M et al. (2017) HER2 and EGFR Overexpression Support Metastatic Progression of Prostate Cancer to Bone. Cancer Res 77(1): 74-85.

43. Bertero E, Ameri P, Maack C (2019) Bidirectional Relationship Between Cancer and Heart Failure: Old and New Issues in Cardio-oncology. Card Fail rev 5(2): 106-111.

44. Agunbiade TA, Zaghlol RY, Barac A (2019) Heart Failure in Relation to Tumor Targeted Therapies and Immunotherapies. Methodist Debakey Cardiovasc J 15(4): 250-257.

45. Negro A, Brar BK, Lee KF (2004) Essential Roles of Her2/erbB2 in Cardiac Development and Function. Recent Prog Horm Res 59: 1-12.

46. Suter TM, Procter M, van Veldhuisen DJ, et al. (2007) Trastuzumabassociated Cardiac Adverse Effects in the Herceptin Adjuvant Trial. J Clin Oncol 25(25): 3859-3865.

47. Jerusalem G, Lancellotti P, Kim SB (2019) HER2+ Breast Cancer Treatment and Cardiotoxicity: Monitoring and Management. Breast Cancer Res Treat 177(2): 237-250.

48. Litvak A, Batukbhai B, Russell SD, Tsai HL, Rosner GL, et al. (208) Racial Disparities in the Rate of Cardiotoxicity of HER2-targeted Therapies Among Women with Early Breast Cancer. Cancer 124(9): 1904-1911.

49. Chang HM, Moudgil R, Scarabelli T, et al. (2017) Cardiovascular Complications of Cancer Therapy: Best Practices in Diagnosis, Prevention, and Management-Part 1. J Am Coll Cardiol 70(20): 25362551.

50. Melincovici CS, Bosca AB, Susman S, et al. (2018) Vascular Endothelial Growth Factor (VEGF) - Key Factor in Normal and Pathological Angiogenesis. Rom J Morphol Embryol 59(2): 455-467.

51. Qi WX, Fu S, Zhang Q, Guo XM (2014) Bevacizumab Increases the Risk of Severe Congestive Heart Failure in Cancer Patients: An Up-to-date Meta-analysis with a Focus on Different Subgroups. Clin Drug Investig 34(10): 681-690.

52. Choueiri TK, Mayer EL, Je Y, Rosenberg JE, Nguyen PL, et al. (2011) Congestive Heart Failure Risk in Patients with Breast Cancer Treated with Bevacizumab. J Clin Oncol 29(6): 632-638.

53. Ghatalia P, Morgan CJ, Je Y, et al. (2015) Congestive Heart Failure with Vascular Endothelial Growth Factor Receptor Tyrosine Kinase Inhibitors. Crit Rev Oncol Hematol 94(2): 228-237.

54. Narayan V, Keefe S, Haas N, et al. (2017) Prospective Evaluation of Sunitinib-induced Cardiotoxicity in Patients with Metastatic Renal Cell Carcinoma. Clin Cancer Res 23(14): 3601-3609.

55. Touyz RM, Herrmann J (2018) Cardiotoxicity with Vascular Endothelial Growth Factor Inhibitor Therapy. NPJ Precis Oncol 2: 13.

56. Suter TM, Ewer MS (2013) Cancer Drugs and the Heart: Importance and Management. Eur Heart J 34(15): 1102-1111.

57. Han X, Zhou Y, Liu W (2017) Precision Cardio-oncology: Understanding the Cardiotoxicity of Cancer Therapy. NPJ Precis Oncol 1(1): 31.

58. Madeddu C, Deidda M, Piras A, et al. (2017) Pathophysiology of Cardiotoxicity Induced by Nonanthracycline Cardiotoxicity. J Cardiovasc Med (Hagerstown) 17 Suppl 1: e12-e18.

59. Sorrentino MF, Kim J, Foderaro AE, Truesdell AG (2012) 5-fluorouracil Induced Cardiotoxicity: Review of the Literature. Cardiol J 19(5): 453458.

60. Rowinsky EK, McGuire WP, Guarnieri T, et al. (1991) Cardiac Disturbances During the Administration of Taxol. J Clin Oncol 9(9): 1704-1712. 
61. Sara JD, Kaur J, Khodadadi R, et al. (2018) 5-fluorouracil and Cardiotoxicity: A Review. Ther Adv Med Oncol 10:1758835918780140.

62. Aghel N, Delgado DH, Lipton JH (2017) Cardiovascular Toxicities of BCR-ABL Tyrosine Kinase Inhibitors in Chronic Myeloid Leukemia: Preventive Strategies and Cardiovascular Surveillance. Vasc Health Risk Manag 13: 293-303.

63. Cole DC, Frishman WH (2018) Cardiovascular Complications of Proteasome Inhibitors Used in Multiple Myeloma. Cardiol Rev 26(3): 122-129.

64. Haguet H, Douxfils J, Chatelain C, et al. (2018) BCR-ABL Tyrosine Kinase Inhibitors: Which Mechanism(s) May Explain the Risk of Thrombosis? TH Open 2(1): e68-e88.

65. Guignabert C, Phan C, Seferian A, et al. (2016) Dasatinib Induces Lung Vascular Toxicity and Predisposes to Pulmonary Hypertension. J Clin Invest 126(9): 3207-3218.

66. Jabbour E, Cortes J, Kantarjian H (2011) Long-Term Outcomes in the Second-Line Treatment of Chronic Myeloid Leukemia: A Review of Tyrosine Kinase Inhibitors. Cancer 117(5): 897-906.

67. Wu P, Oren O, Gertz MA, Yang EH (2020) Proteasome Inhibitor-Related Cardiotoxicity: Mechanisms, Diagnosis, and Management. Curr Oncol Rep 22(7): 66.

68. Shah C, Bishnoi R, Jain A, Bejjanki H, Xiong S, et al. (2018) Cardiotoxicity Associated with Carfilzomib: Systematic Review and Meta-Analysis Leuk Lymph 59(11): 2557-2569.

69. Shoukat S, Zheng D, Yusuf SW (2019) Cardiotoxicity Related to Radiation Therapy. Cardiol Clin 37(4): 449-458.

70. da Silva RMFL (2019) Effects of Radiotherapy in Coronary Artery Disease. Curr Atheroscler Rep 21(12): 50.

71. Liu LK, Ouyang W, Zhao X, et al. (2017) Pathogenesis and Prevention of Radiation-induced Myocardial Fibrosis. Asian Pac J Cancer Prev 18(3): 583-587.

72. Welch TD (2018) Constrictive Pericarditis: Diagnosis, Management and Clinical Outcomes. Heart. 104(9): 725-731.

73. Saiki H, Moulay G, Guenzel AJ, Liu W, Decklever TD, et al. (2013) Experimental Cardiac Radiation Exposure Induces Ventricular Diastolic Dysfunction with Preserved Ejection Fraction. Am J Physiol
Heart Circ Physiol 313(2): H392-H407.

74. Shenoy S, Shetty S, Lankala S, et al (2017) Cardiovascular Oncologic Emergencies. Cardiology 138(3): 147-158.

75. Warren WH (2000) Malignancies Involving the Pericardium. Semin Thorac Cardiovasc Surg 12(2): 119-129.

76. Brunetti ND, Tarantino N, Guastafierro F, De Gennaro L, Correale M, et al. (2019) Malignancies and Outcome in Takotsubo Syndrome: A MetaAnalysis Study on Cancer and Stress Cardiomyopathy. Heart Fail Rev 24(4): 481-488.

77. da Silva Costa IBS, Figueiredo CS, Fonseca SMR, Bittar CS, de Carvalho Silva CMD, et al. (2019) Takotsubo Syndrome: An Overview of Pathophysiology, Diagnosis and Treatment with Emphasis on Cancer Patients. Heart Fail Rev 24(6): 833-846.

78. Templin C, Ghadri JR, Diekmann J, Napp LC, Bataiosu DR, et al. (2015) Clinical Features and Outcomes in Takotsubo (stress) Cardiomyopathy. N Engl J Med 373(10): 929-938.

79. Jin C, Sharma AN, Thevakumar B, Majid M, Al Chalaby S, et al. (2020) Carcinoid Heart Disease: Pathophysiology, Pathology, Clinical Manifestations, and Management. Cardiology 16: 1-9.

80. Connolly HM, Schaff HV, Mullany CJ, Rubin J, Abel MD, et al. (2001) Surgical Management of Left-Sided Carcinoid Heart Disease. Circulation 104 (12 Suppl 1): I36-140.

81. Zhai LR, Zhang XW, Yu T, Jiang ZD, Huang DW, et al. (2020) Primary Ovarian Carcinoid: Two Cases Report and Review of the Literature. Medicine (Baltimore) 99(40): e21109.

82. Perry D, Hayek SS (2019) Carcinoid Heart Disease: A Guide for Clinicians. Cardiol Clin 37(4): 497-503.

83. Gertz MA (2020) Immunoglobulin Light Chain Amyloidosis: 2020 Updated on Diagnosis, Prognosis, and Treatment 95(7): 848-860.

84. Witteles RM, Liedtke M (2019) AL Amyloidosis for the Cardiologist and Oncologist: Epidemiology, Diagnosis, and Management. J Am Coll Cardiol 1(1): 117-130.

85. Kattih B, Shirvani A, Klement P, Garrido AM, Gabdoulline R, et al. (2020) IDH1/2 Mutations in Acute Myeloid Leukemia Patients and Risk of Coronary Artery Disease and Cardiac Dysfunction - A Retrospective Propensity Score Analysis. Leukemia.

\section{Your next submission with Juniper Publishers will reach you the below assets}

- Quality Editorial service

- Swift Peer Review

- Reprints availability

- E-prints Service

- Manuscript Podcast for convenient understanding

- Global attainment for your research

- Manuscript accessibility in different formats

(Pdf, E-pub, Full Text, Audio)

- Unceasing customer service

Track the below URL for one-step submission

https://juniperpublishers.com/online-submission.php 